\title{
The HdpH DSLs for Scalable Reliable Computation
}

\author{
Patrick Maier \\ University of Glasgow \\ Patrick.Maier@glasgow.ac.uk
}

\author{
Robert Stewart \\ Heriot-Watt University \\ R.Stewart@hw.ac.uk
}

\author{
Phil Trinder \\ University of Glasgow \\ Phil.Trinder@glasgow.ac.uk
}

\begin{abstract}
The statelessness of functional computations facilitates both parallelism and fault recovery. Faults and non-uniform communication topologies are key challenges for emergent large scale parallel architectures. We report on $H d p H$ and $H d p H-R S$, a pair of Haskell DSLs designed to address these challenges for irregular task-parallel computations on large distributed-memory architectures. Both DSLs share an API combining explicit task placement with sophisticated work stealing. $\mathrm{HdpH}$ focuses on scalability by making placement and stealing topology aware whereas HdpH-RS delivers reliability by means of fault tolerant work stealing.

We present operational semantics for both DSLs and investigate conditions for semantic equivalence of $\mathrm{HdpH}$ and $\mathrm{HdpH}-\mathrm{RS}$ programs, that is, conditions under which topology awareness can be transparently traded for fault tolerance. We detail how the DSL implementations realise topology awareness and fault tolerance. We report an initial evaluation of scalability and fault tolerance on a 256-core cluster and on up to $32 \mathrm{~K}$ cores of an HPC platform.
\end{abstract}

Categories and Subject Descriptors D.3.3 [Programming Languages]: Language Constructs and Features

Keywords embedded domain specific 1 anguages; parallelism; topology awareness; fault tolerance

\section{Introduction}

As the manycore revolution drives up the number of cores, the use of compute resources with $100,000+$ cores will become commonplace in the near future. On such architectures core failures are predicted to become relatively common [3, 6]. Moreover, at such scales core-to-core communication latency may vary by several orders of magnitude, depending on whether the communicating cores belong to different compute nodes, possibly living in different subnets, or whether they share memory or cache.

Reliability and topology awareness are substantial challenges even for applications that are embarrassingly parallel, or have other simplifying properties like regular static task decomposition, or simple data structures. The challenges are yet greater for applications without these properties. Symbolic computations, for example, often have highly irregular task granularity, dynamic task creation, complex control flows, or complex data structures. (c) ACM, 2014. This is the author's version of the work. It is posted here by permission of ACM for your personal use. Not for redistribution. The definitive version was published in Haskell '14: Proceedings of the 2014 ACM SIGPLAN symposium on Haskell, (2014) http://doi.acm.org/10.1145/10.1145/2633357.2633363
We present $H d p H$ (Haskell distributed parallel Haskell) and HdpH-RS (HdpH + Reliable Scheduling), a pair of DSLs designed to address the challenges of reliability and scalability for computations with irregular parallelism. Specifically $\mathrm{HdpH}$ allows the programmer to exploit communication topologies, and HdpH-RS provides low cost automatic fault tolerance. The languages were developed for symbolic computation on capability class highperformance computing (HPC) platforms (currently around $10^{5}$ cores) and on commodity off-the-shelf (COTS) platforms, but have broad application for large-scale irregularly-parallel computations.

We start by outlining related work on parallel languages/runtimes and parallel symbolic computation (Section 2. We present the design of $\mathrm{HdpH}$ that is novel in combining the following features (Section 3. It is scalable, providing a distributed-memory parallel DSL that manages computations on multiple multicore nodes. It provides high-level semi-explicit parallelism with implicit and explicit task placement, and dynamic load management by work stealing. It provides semi-explicit topology awareness allowing the programmer to exploit the deep communication topology of large architectures using an abstract distance metric (Section 3.2. HdpH-RS features a reliable scheduler to provide low cost automatic fault tolerance using Erlang-style supervision and recovery of location-invariant computations (Section 3.4). $\mathrm{HdpH}-$ RS currently omits topology awareness, but this is an engineering artifact rather than a fundamental issue. Both $\mathrm{HdpH}$ and $\mathrm{HdpH}-\mathrm{RS}$ provide high-level coordination abstractions via polymorphic algorithmic skeletons (Section77).

The initial $\mathrm{HdpH}$ and $\mathrm{HdpH}-\mathrm{RS}$ designs are reported in [25]. The following are novel research contributions.

(1) We present operational semantics for $\mathrm{HdpH}$ and $\mathrm{HdpH}-\mathrm{RS}$. The semantics combines non-deterministic scheduling of parallelism with topology awareness and fault tolerance. We investigate conditions for semantic equivalence of $\mathrm{HdpH}$ and $\mathrm{HdpH}-\mathrm{RS}$ programs to enable trading topology awareness for fault tolerance (Section 4).

(2) We outline the $\mathrm{HdpH}$ and $\mathrm{HdpH}-\mathrm{RS}$ implementations, focusing on how the work stealing schedulers achieve topology awareness and fault tolerance (Section 6), to implement the operational semantics. This includes validating HdpH-RS' sophisticated fault tolerant work stealing protocol by model checking (Section 5).

(3) We present an initial evaluation of $\mathrm{HdpH}$ and $\mathrm{HdpH}-\mathrm{RS}$ on a 256-core COTS cluster and on HECToR, a capability class HPC platform with a total of 90,000 cores. We demonstrate the scalability of both $\mathrm{HdpH}$ and $\mathrm{HdpH}-\mathrm{RS}$. We investigate the fault tolerance of HdpH-RS, and the overheads both in the presence and absence of faults. We sketch a case study using $\mathrm{HdpH}$ to coordinate 1000 instances of the GAP computer algebra system [10] on HECToR to solve problems in algebraic representation theory (Section 8 .

\section{Related Work}

Parallel sublanguages. Most production languages have multiple parallel or distributed sub-languages that may be built-in to 


\begin{tabular}{|c|c|c|c|c|c|c|c|}
\hline & \multicolumn{3}{|c|}{ Built-In } & \multicolumn{4}{|c|}{ DSLs } \\
\hline $\begin{array}{ll} & \text { Language } \\
\text { Property } & \end{array}$ & $\begin{array}{l}\text { GpH } \\
\text { GUM }\end{array}$ & Eden & GHC & $\begin{array}{l}\text { Par } \\
\text { monad }\end{array}$ & $\begin{array}{l}\text { Cloud } \\
\text { Haskell }\end{array}$ & $\begin{array}{l}\text { Meta } \\
\text { Par }\end{array}$ & $\begin{array}{c}\mathrm{HdpH} \\
\text { (RS) }\end{array}$ \\
\hline Scalable - dist. mem. & + & + & - & - & + & + & + \\
\hline Fault Tolerance & - & - & - & - & + & - & + \\
\hline Polymorphic Closures & + & + & + & + & - & - & + \\
\hline Pure (non-monad) API & + & + & + & - & - & - & - \\
\hline Determinism & $(+)$ & $(+)$ & $(+)$ & + & - & + & - \\
\hline Auto Load Balancing & + & + & + & + & - & + & + \\
\hline
\end{tabular}

Table 1. Parallel Haskell comparison.

the language implementation like OpenMP or built-on like MPI libraries. Recently DSL technology is being exploited to build parallel or distributed sub-languages onto existing languages. For example the Akka toolkit [1] that provides Erlang-style distributed actors for Scala and Java can be viewed as a shallowly-embedded DSL.

In addition to standard DSL support capabilities like higherorder functions and meta-programming, parallel/distributed DSLs require to manipulate computations, often expressed as futures or closures. Haskell augments these capabilities with a non-strict semantics that minimises sequentialisation and makes it relatively easy to provide a Monad to specify the required parallel or distributed coordination behaviour.

Parallel Haskells. Haskell language extensions like Eden [20], $\mathrm{GpH}$ [33] and GHC [26] build in parallelism in the form of elaborate runtime systems (RTS) that support parallelism primitives. To improve maintainability and ease development several recent parallel Haskells are monadic DSLs embedded in Concurrent Haskell [29], e. g. CloudHaskell [8], the Par monad [27], MetaPar [9] and our new languages HdpH and HdpH-RS. Table 1 compares the key features of some important general purpose parallel Haskells, excluding more specialised variants like Data Parallel Haskell [7]. Most of the entries in the table are self-explanatory. The determinism properties of these languages are not trivial [16], and here we mean that the language guarantees that parallel evaluation does not introduce observable concurrency, e. g. due to races between parallel threads ${ }^{1}$

The crucial differences between $\mathrm{HdpH} / \mathrm{HdpH}-\mathrm{RS}$ and other parallel Haskells can be summarised as follows. Both GHC and the Par monad provide parallelism only on a single multicore, where $\mathrm{HdpH}$ scales onto distributed-memory architectures with many multicore nodes. Meta-Par focuses on exploiting heterogeneous, rather than the relatively homogeneous HPC platforms that $\mathrm{HdpH} / \mathrm{HdpH}-\mathrm{RS}$ target. CloudHaskell replicates Erlang style [2] explicit distribution and is the only other Haskell variant to provide fault tolerance. It is most closely related to $\mathrm{HdpH}$, but provides lower level coordination with explicit task placement and no load management. As CloudHaskell distributes only monomorphic closures it is not possible to construct general coordination abstractions like algorithmic skeletons.

Topology aware and fault tolerant scheduling are novel features of the HdpH/HdpH-RS DSLs. Topology aware work stealing in HotSLAW [28] and load balancing in CHARM++ [17] minimise the cost of task migration but do not expose the topology to the programmer, and hence unlike $\mathrm{HdpH}$ cannot guarantee that tasks remain close to each other. While some GRID/cloud middleware like [15] exposes complex topologies, the architectures are very different from HPC and the schedulers typically aim to minimise the cost of inter-process communication rather than migration. Perhaps most closely related is a parallel Haskell [14] that exposes a two-level topology. In contrast $\mathrm{HdpH}$ topologies may be arbitrarily deep.

\footnotetext{
${ }^{1}$ GUM, Eden and GHC guarantee determinism only for pure computations.
}

Erlang [2] fault tolerance links processes, and supervision trees are commonly constructed where one process supervises others, that may in turn be supervisors. The supervisor is informed of the failure of any supervised process and takes actions like respawning the failed process or killing sibling processes. Unlike HdpH-RS the supervised processes are stateful and hence recovery is observable; moreover recover policies are explicitly stated for each supervisor. Distributed query frameworks like Google MapReduce or Hadoop [34] provide automatic recovery of read-only, and hence idempotent, functions. Unlike $\mathrm{HdpH}-\mathrm{RS}$ the programming model provided by these frameworks is a restricted to distributed data retrieval. Both Erlang and Hadoop tasks are placed only once, simplifying replication and recovery. In contrast $\mathrm{HdpH}-\mathrm{RS}$ must recover sparks that may have migrated to a new location since their initial placement.

General purpose fault tolerant work stealing is a relatively unexplored area. Some closely related work is [21] that provides task parallel fault tolerant scheduling of idempotent computations with work stealing. Satin [35] uses a global result table for sharing computation values to limit re-computation in the presence of failure, and Cilk-NOW [4] that checkpoints individual computations to allow available schedulers to resume partially executed computations in the presence of failure.

Symbolic computation and GAP. Symbolic computation is key to both mathematics and computer science, e. g. for cryptography. Computational algebra is an important class of symbolic computation with many complex and expensive computations that would benefit from parallel execution. Besides well-known generalpurpose Computational Algebra Systems (CAS) like Maple, there are a number of CAS specialised to particular mathematical domains, e. g. GAP [10] to combinatorial group theory.

Parallel symbolic computation. Some discrete mathematical problems are embarrassingly parallel, and this has been exploited for years even at Internet scale, e.g. the "Great Internet Mersenne Prime Search". Other problems have more complex coordination patterns and both parallel algorithms and parallel CAS implementations have been developed, e. g. ParGAP. Many parallel algebraic computations exhibit high degrees of irregularity, with varying numbers and sizes of tasks. Some computations have both multiple levels of irregularity, and enormous ( 5 orders of magnitude) variation in task sizes [18]. They use complex user-defined data structures and have complex control flows, often exploiting recursion. They make little, if any, use of floating-point operations.

This combination of characteristics means that symbolic computations are not well suited to conventional HPC paradigms with their emphasis on iteration over matrices of floating point numbers, and has motivated the development of domain specific scheduling and management frameworks like SymGridPar [18].

SymGridPar, SymGridPar2 and HdpH. The SymGridPar framework [18] is a client/server infrastructure for orchestrating multiple CAS instances into a parallel application. To the user it presents itself as a set of algorithmic skeletons for introducing parallelism, embedded into the user's CAS (the client). The skeletons are implemented in a CAS-independent distributed middleware (the coordination server), which performs load balancing and calls other CAS (the compute servers) via remote procedure call.

SymGridPar2 (SGP2) is a successor to SymGridPar that aims to scale symbolic computation to architectures with $10^{5}$ cores. The SGP2 design aims to preserve the user experience of SGP, specifically the high-level skeleton API. That is, to the CAS user SGP2 will look like SGP, apart from a few new skeleton parameters for tuning locality control and/or fault tolerance. SGP2 retains the architecture of SGP but provides a scalable fault tolerant coordination server. A key design decision is to realise the coordination server 
using the $\mathrm{HdpH}$ and $\mathrm{HdpH}-\mathrm{RS}$ DSLs that are the focus of this paper.

Faults in large-scale architectures. HPC architectures exploit extremely reliable processor and interconnect technologies and current system still exhibit low fault rates. However, fault rates grow rapidly with the number of cores. In consequence fault tolerance for large HPC architectures is a very active research area [6]. We know from warehouse computing that fault rates are likely to be far greater with the much cheaper and more prevalent commodity-offthe-shelf (COTS) architectures [3].

\section{Language Design}

This section presents the designs of $\mathrm{HdpH}$ and $\mathrm{HdpH}-\mathrm{RS}$, shallowly embedded Haskell DSLs for semi-explicit parallelism on large distributed-memory platforms. The DSLs have the following novel combination of features. They are scalable, each providing a parallel DSL for distributing computations across a network of multicore nodes. They are portable, being implemented entirely in Haskell (with GHC extensions) rather than relying on bespoke lowlevel runtime systems like Glasgow parallel Haskell $(\mathrm{GpH})[32]$ or Eden [20]. HdpH and HdpH-RS provide high-level semi-explicit parallelism with implicit and explicit task placement and dynamic load management. Implicit placement frees the programmer from coding work distribution and load management. Instead, idle nodes steal work from busy nodes automatically, thereby maximising utilisation when there is enough work to be stolen at the expense of deterministic execution (Section 3.3. HdpH focuses on semiexplicit topology awareness allowing the programmer to exploit the deep communication topology of large architectures using an abstract distance metric (Section 3.2). HdpH-RS provides low cost automatic fault tolerance using Erlang-style supervision and recovery of location-invariant computations (Section 3.4). Switching between topology awareness and fault tolerance comes at minimal cost as both DSLs share the same polymorphic API (Section 3.1). Polymorphism is also a key feature of advanced coordination $\mathrm{ab}$ stractions such as algorithmic skeletons combining explicit and implicit task placement (Section 7).

\subsection{Primitives}

HdpH extends the Par monad DSL [27] for shared-memory parallelism to distributed memory, and Figure 1 lists the HdpH API. $\mathrm{HdpH}$ exposes locations and distances between locations as abstract types Node and Dist. The functions dist and equiDist provide information about nodes and distances as detailed in Section 3.2

Like [27], $\mathrm{HdpH}$ focuses on task parallelism. In distributed memory, this requires serialisation of Par computations and results so they can be sent over the network. While the Binary typeclass provides serialisation of evaluated values (normal forms), computations (thunks) must be wrapped into explicit closures. An explicit closure is a term of type Closure $t$, which wraps a possibly unevaluated value of type $t$. Generalising CloudHaskell's closures [8], the explicit closures of $\mathrm{HdpH}$ are fully polymorphic as there is no constraint on the type parameter $t$; this is crucial for building general purpose coordination abstractions like polymorphic skeletons (Section 7) with the $\mathrm{HdpH}$ primitives.

$\mathrm{HdpH}$ provides the following closure primitives: unClosure unwraps a Closure $t$ and returns its value of type $t$; toClosure wraps a normal form of any serialisable type $t$, i.e. any type which an instance of Binary, into a Closure t. To construct explicit closures by wrapping thunks, including of types that cannot have Binary instances like Par computations, HdpH offers a Template Haskell macro for explicit closure conversion. More precisely, the Template Haskell splice $\$(\mathrm{mkClosure}[|\mathrm{e}|]$ ) constructs a

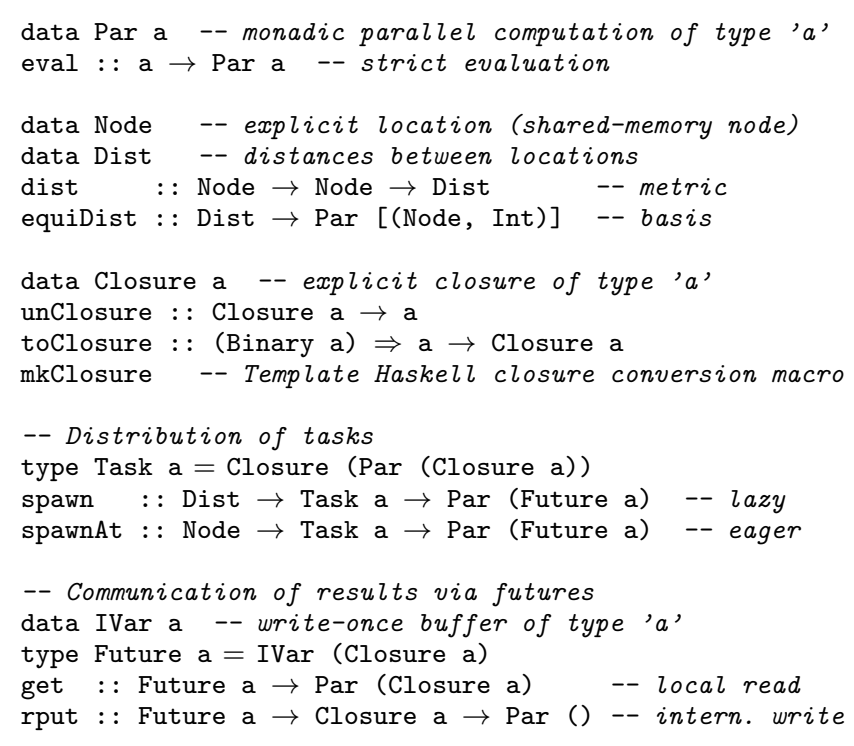

Figure 1. Types and primitives of $\mathrm{HdpH}$ and $\mathrm{HdpH}-\mathrm{RS}$.

Closure $t$ wrapping the unevaluated thunk e of type $t$, provided the captured free variables of e are serialisable; see [23] for details.

In $\mathrm{HdpH}$, a task computing a value of type $\mathrm{t}$ is an expression of type Closure (Par (Closure $t$ )), i.e. a serialisable monadic computation that will deliver a serialisable value of type $t$. $\mathrm{HdpH}$ offers two task distribution primitives, spawn and spawnAt, the scheduling of which is discussed below (Section 3.3). Both primitives immediately return a future [12] of type IVar (Closure $t$ ). Such an IVar is a write-once buffer expecting the result of the task, which is an explicit closure of type $t$. The actual result can be read by calling get, blocking until the result is available. Note that a future is not serialisable, hence cannot be captured by explicit closures. As a result the future can only be read on the hosting node, i. e. the node it was created on. The internal primitive rput ${ }^{2}$ transparently writes to a remote future, regardless where it is hosted, and silently fails if the future is already full or the host is dead.

The example below illustrates the use of the $\mathrm{HdpH}$ primitive ${ }^{3}$ to sum the Liouville function [5] from 1 to $n$ in parallel. The code shows how to construct a list of tasks with the mkClosure macro, how to generate parallelism by spawning the tasks (the distance argument 1 will be explained later), how to retrieve the results closures, and how to unwrap them and return the final sum.

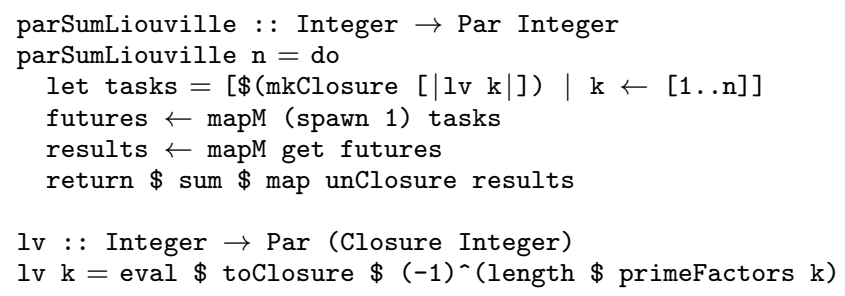

\subsection{Distance Metric and Equidistant Bases}

$\mathrm{HdpH}$ takes an abstract view of the network topology, modelling it as a hierarchy, i. e. an unordered tree whose leaves correspond to compute nodes, as in Figure 2 for instance. Every subtree of the hierarchy forms a virtual cluster. The interpretation of these

\footnotetext{
${ }^{2}$ One cannot call rput directly; it is used only by scheduler and semantics.

${ }^{3}$ Parallel map skeletons (Section 7 provide a more elegant solution.
} 


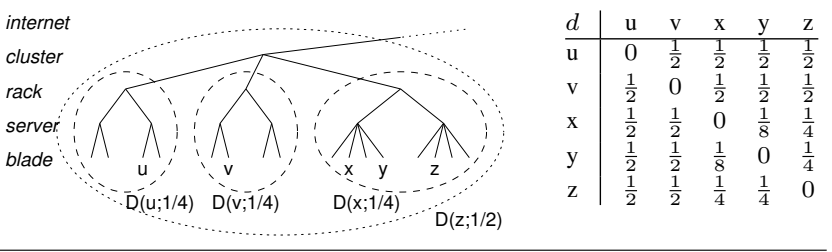

Figure 2. Hierarchy, distance metric and equidistant partition.

virtual clusters is not fixed; e.g. Figure 2 suggests a cluster, possibly connected to others over the Internet, consisting of multiple racks, which in turn house several servers, each containing multiple blades. The hierarchy need not exactly reflect the physical network topology. Rather, it presents a logical arrangement of the network into virtual clusters of manageable size. However, actual latencies should be reasonably compatible, i.e. in general the latency within a virtual cluster should be no higher than the latency between sibling clusters.

Such hierarchies can be represented concisely by a distance function $d$ on nodes that is defined by

$$
d(p, q)= \begin{cases}0 & \text { if } p=q \\ 2^{-n} & \text { if } p \neq q \text { and } n=\text { length of longest } \\ & \text { common path from root to } p \text { and } q .\end{cases}
$$

Figure 2 tables sample distances corresponding to the hierarchy. Mathematically, $d$ defines an ultrametric space on the set of nodes. That is, $d$ is non-negative, symmetric, 0 on the diagonal, and satisfies the strong triangle inequality: $d\left(p_{1}, p_{3}\right) \leq$ $\max \left\{d\left(p_{1}, p_{2}\right), d\left(p_{2}, p_{3}\right)\right\}$ for all nodes $p_{1}, p_{2}, p_{3}$.

Given a node $p$ and $r \geq 0$, define $D(p ; r)=\{q \mid d(p, q) \leq r\}$ to be the ball with centre $p$ and radius $r$. Balls correspond to virtual clusters in the hierarchy, see Figure 2 for a few examples. Balls have the following properties, thanks to $d$ being an ultrametric.

1. Every node inside a ball is its centre.

2. Every ball of radius $r=2^{-n}$ is uniquely partitioned by a set of balls of radius $\frac{1}{2} r$, the centres of which are pairwise spaced distance $r$ apart.

We call the set $\left\{D\left(q ; \frac{1}{2} r\right) \mid q \in D(p ; r)\right\}$ the equidistant partition of $D(p ; r)$. A set $Q$ of nodes is an equidistant basis for $D(p ; r)$ if $Q$ contains exactly one centre of each ball in the equidistant partition of $D(p ; r)$. Due to property 1 equidistant bases are not unique. To illustrate, Figure 2 shows the equidistant partition of $D\left(z ; \frac{1}{2}\right)$, from which we can read off that $\{u, v, x\}$ and $\{u, v, y\}$ are two equidistant bases.

$\mathrm{HdpH}$ reifies the metric $d$ as the pure function dist, and this implies that all nodes agree on the metric, and that the metric cannot change over time. The primitive equiDist takes a radius $r$ and returns a size-enriched equidistant basis for $D\left(p_{0} ; r\right)$, where $p_{0}$ is the current node. More precisely, equiDist returns a non-empty list $\left[\left(q_{0}, n_{0}\right),\left(q_{1}, n_{1}\right), \ldots\right]$ such that the $q_{i}$ form an equidistant basis for $D\left(p_{0} ; r\right)$ and $n_{i}$ is the size of $D\left(q_{i} ; \frac{1}{2} r\right)$. By convention, $q_{0}$ is the current node $p_{0}$, so the current node can be queried thus:

myNode : : Par Node

myNode $=$ do $\left\{\left(\left(\mathrm{p},{ }_{-}\right):_{-}\right) \leftarrow\right.$ equiDist 0 ; return $\left.\mathrm{p}\right\}$

The operator allNodes : : Par [Node] for computing a list of all known nodes is also expressible in terms of equiDist as a recursive distributed gather operation.

\subsection{Scheduling}

Lazy, implicit task placement. The spawn primitive places a task into a local task pool, from where it is scheduled by on-demand work stealing, either locally or onto a remote node looking for work. Crucially, work stealing is non-deterministic, which makes $\mathrm{HdpH}$ a non-deterministic DSL because location-awareness, e. g. via calls to myNode, may reveal scheduling decisions.

The first argument to spawn is the task radius $r$ that constrains how far the task can travel from the spawning node $p_{0}$ : it can be scheduled precisely by the nodes in the ball $D\left(p_{0} ; r\right)$. The extreme radii deserve special attention: $r=1$ means the task may be scheduled on any node, and $r=0$ means the task cannot be scheduled anywhere but $p_{0}$.

The key features of topology aware on-demand scheduling are as follows, and their implementation is outlined in Section 6.2 No task is ever scheduled beyond its task radius. Tasks with small radii are preferred for local execution. Tasks with big radii are preferably scheduled far away, depending on demand.

Eager, explicit task placement. Scheduling tasks on demand by random work stealing performs well with irregular parallelism. However, it tends to under-utilise large scale architectures at the beginning of the computation. To combat this drawback, HdpH complements spawn with spawnAt, which places a task on a named node where it is scheduled for execution immediately, taking priority over any implicitly placed tasks. Eager execution implies that the task is meant to perform coordination, e. g. spawn further tasks, rather than actual computation.

\subsection{Fault Tolerance}

Crucially each $\mathrm{HdpH}$ node's heap is isolated from the heaps of other nodes. Hence the failure of one node does not poison computations on other nodes. HdpH-RS provides automatic fault tolerance using Erlang style supervision and recovery of locationinvariant computations, that is computations that always produce the same effect regardless where they are executed (Section 4 . Compared to other languages, fault tolerance in HdpH-RS is relatively sophisticated: for example when Erlang [2] and Hadoop [34] place tasks on remote nodes, these tasks do not move. This simplifies replication and recovery, whereas HdpH-RS provides replication and recovery even when computations migrate between nodes.

Reliable scheduling. Fault tolerance in HdpH-RS is provided by replacing the $\mathrm{HdpH}$ scheduler with a reliable scheduler that handles failures automatically. The core of the reliable scheduler is an alternative work stealing protocol that enables supervisors to track the locations of tasks. In HdpH-RS, a supervisor is a future created by spawn or spawnAt. As long as it is empty, a supervising future stores a backup copy of the spawned task and monitors the health of all nodes potentially holding the task. The reliable scheduler will recover tasks lost due to node failure by replicating the backups stored in supervising futures elsewhere, ensuring that all futures are eventually filled. The implementation of the $\mathrm{HdpH}-$ RS scheduler is sketched in Section 6.3, a complete exposition of the design and implementation can be found in the thesis [30].

\section{Operational Semantics}

This section presents an operational semantics for $\mathrm{HdpH}$ and HdpH-RS in the style of [27], focusing on topology aware scheduling and fault recovery. Figure 3 introduces the syntax of terms and values. The language is essentially the same as the embedded DSL presented in Section 3 , except that the semantics ignores explicit closures, i. e. assumes that all terms are implicitly serialisable. However, the semantics does restrict the second arguments of spawn, spawnAt and rput to terms $M$ such that $f n(M)=\emptyset$, i. e. terms not containing free (names of) IVars; this is justified because in Section 3 these arguments are explicit closures, which cannot capture free IVars. For simplicity, the semantics also treats the pure function dist as a monadic primitive. 


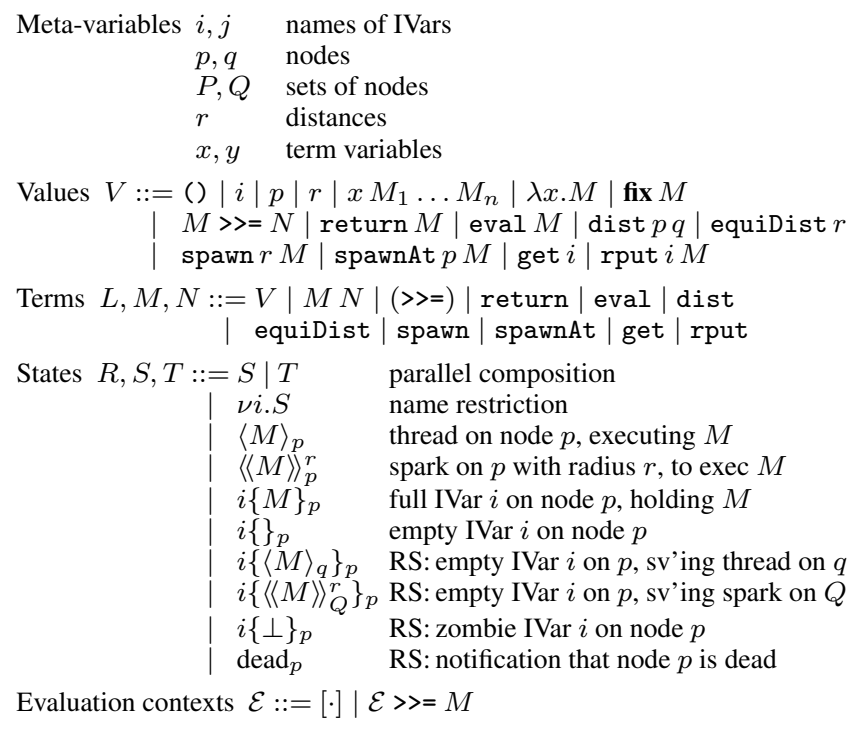

Figure 3. Syntax of $\mathrm{HdpH}$ and $\mathrm{HdpH}-\mathrm{RS}$ terms, values and states.

$$
\begin{array}{crr}
S|T \equiv T| S & \nu i . \nu j . S & \equiv \nu j . \nu i . S \\
R|(S \mid T) \equiv(R \mid S)| T \quad \nu i .(S \mid T) & \equiv(\nu i . S) \mid T, \quad i \notin f n(T) \\
\frac{S{ }_{d} T}{R\left|S \longrightarrow_{d} R\right| T} & \frac{S \longrightarrow{ }_{d} T}{\nu i . S \longrightarrow_{d} \nu i . T} & \frac{S \equiv S^{\prime} \longrightarrow_{d} T^{\prime} \equiv T}{S \longrightarrow{ }_{d} T}
\end{array}
$$

Figure 4. Structural congruence and structural transitions.

For the purposes of the DSL semantics, the host language is a standard lambda calculus with fixed points and some data constructors for nodes, distances, integers and lists (omitted to save space). We assume a big-step operational semantics for the host language, and write $M \Downarrow V$ to mean that there is a derivation proving that term $M$ evaluates to value $V$. The definition of the big-step semantics is entirely standard (and omitted). Note that the syntax of values in Figure 3 implies that the DSL primitives are strict in arguments of type Node, Dist and IVar.

\subsection{Semantics of HdpH}

The operational semantics of the HdpH DSL is a small-step reduction semantics $\longrightarrow_{d}$ indexed by a distance metric $d$. The reduction relation operates on the states defined in Figure 3 A state is built from atomic states by parallel composition and name restriction. Each atomic state has a location indicated by the subscript $p$. An atomic state of the form $\langle M\rangle_{p}$ or $\langle\langle M\rangle\rangle_{p}^{r}$, where $M$ is a computation of type Par (), denotes a thread or spark, respectively; sparks differ from threads in that they may migrate within radius $r$ around their current node $p$. An atomic state of the form $i\{?\}_{p}$ denotes an IVar named $i$; the place holder "?" signals that we don't care whether $i$ is empty or full. Figure 4 asserts the usual structural congruence properties of parallel composition and name restriction, and the usual structural transitions propagating reduction under parallel composition and name restriction.

Figure 5 presents the transition rules for $\mathrm{HdpH}$. Most of these rules execute a thread, relying on an evaluation context $\mathcal{E}$ to select the first action of the thread's monadic computation. Rules that are similar to those in [27] are not explained in detail.

The first three rules are standard for monadic DSLs; note how eval is just a strict return. The rules (spawn) and (spawnAt) define the work distribution primitives. The primitive spawn creates an IVar $i$ on the current node $p$ and wraps its argument $M$, followed by a write to $i$, into a spark residing on $p$ and bounded by radius $r$. In contrast, spawnAt wraps $M$ into a thread, which is placed on node $q$. The side condition on both rules ensures that the name $i$ is fresh, i. e. does not occur free in the current thread. The rules for IVars are similar to those in [27] except that IVars in HdpH can only be read on the node they reside on. They can however be written from any node, and writes can be raced ${ }^{4}$ the first write wins, subsequent writes have no effect. The rules (dist) and (equiDist) define the eponymous topology aware primitives. These two rules, and the spark migration rule, are the only ones that actually require the distance metric $d$.

Rules (migrate) and (convert) govern the scheduling of sparks. A spark may migrate from node $p$ to $q$, provided the distance between the two is bounded by the spark's radius $r$. Sparks cannot be executed directly; instead they must be converted into threads that can execute but not migrate. The $\left(\mathrm{gc}_{-} *\right)$ rules eliminate garbage, i. e. terminated threads and inaccessible IVars. Note that to become garbage, IVars must be filled and sparks must be converted and executed to termination.

We call a thread $\langle M\rangle_{p}$ reachable from a state $S$ iff there is a state $T$ such that $S \longrightarrow_{d}^{*} \nu i_{1} \ldots \nu i_{n} .\left(T \mid\langle M\rangle_{p}\right)$, where $\longrightarrow_{d}^{*}$ denotes the reflexive-transitive closure of $\longrightarrow_{d}$. We call state $S$ well-formed iff there is a root thread $\langle M\rangle_{p}$ with $f n(M)=\emptyset$ such that $\langle M\rangle_{p} \longrightarrow_{d}^{*} S$. We observe that $\longrightarrow_{d}$ reductions starting from well-formed states cannot get stuck except when embedding the host language, namely term $M$ diverging in rules (normalize) and (eval). In particular, well-formedness guarantees that all rputs find their target IVars, that all gets find their source IVars, and that these source IVars are hosted locally.

\subsection{Fault Tolerant Semantics of HdpH-RS}

The operational semantics of HdpH-RS $\stackrel{\mathrm{RS}}{\longrightarrow}$ is an extension of $\longrightarrow_{d}$, i. e. it is a small-step reduction relation on states defined by the same rules, with some small adaptions and some additions.

To model supervision, empty IVars $i\left\{\langle M\rangle_{q}\right\}_{p}$ and $j\left\{\langle\langle N\rangle\rangle_{Q}^{r}\right\}_{p}$ are annotated with the thread $M$ resp. spark $N$ that is supposed to fill them and with some knowledge of the current location of $M$ resp. $N$. In case of non-migratable thread $M$ that knowledge is the node $q$ where $M$ was scheduled by spawnAt. In case of spark $N$, however, the supervisor may not know the actual node due to migration, hence $i$ is annotated with a set of nodes $Q$ overapproximating the true location of $N$ (or of the thread that $N$ has been converted to).

To model node failure, we add atomic states $\operatorname{dead}_{p}$, signalling that node $p$ has died, and $i\{\perp\}_{p}$, representing a zombie IVar $i$, i. e. an effectively dead IVar $i$ on a dead node $p$. The four rules in the top right corner of Figure 6 define the fault model of HdpH-RS. A node $p$ may die any time, signalled by the spontaneous production of dead $_{p}$, and non-deterministically its sparks and threads may disappear and its IVars may turn into zombies. IVars cannot just disappear, or else writes to IVars on dead nodes would get stuck instead of behaving like no-ops. However, some of $p$ 's sparks and threads may survive and continue to execute. In this way the semantics models partial faults and pessimistic notification of faults. Node failure is permanent as no transition consumes dead ${ }_{p}$.

The remaining rules in Figure 6 are the new/adapted transitions for HdpH-RS. Rules (rput_empty_thread) and (rput_empty_spark) fill empty supervising IVars. Rule (rput_zombie) lets remote writes to zombie IVars to fail silently, and (gc_zombie) garbage collects inaccessible zombie IVars. The rules (spawn) and (spawnAt) are identical to the $\mathrm{HdpH}$ rules except for remembering the new

\footnotetext{
${ }^{4}$ Since the DSL in Section 3 does not expose rput, races only occur as a result of task replication in HdpH-RS.
} 


$$
\begin{aligned}
& \langle\mathcal{E}[M]\rangle_{p} \longrightarrow{ }_{d}\langle\mathcal{E}[V]\rangle_{p}, \quad \text { if } M \Downarrow V \text { and } M \not \equiv V \\
& \langle\mathcal{E}[\operatorname{return} N>\rangle=M]\rangle_{p} \longrightarrow_{d}\langle\mathcal{E}[M N]\rangle_{p} \\
& \langle\mathcal{E}[\text { eval } M]\rangle_{p} \longrightarrow_{d}\langle\mathcal{E}[\text { return } V]\rangle_{p}, \quad \text { if } M \Downarrow V \\
& \left.\left.\langle\mathcal{E}[\text { spawn } r M]\rangle_{p} \longrightarrow_{d} \nu i .\left(\langle\mathcal{E}[\text { return } i]\rangle_{p}\left|i\{\}_{p}\right|\langle\langle M\rangle\rangle=\text { rput } i\right\rangle\right\rangle_{p}^{r}\right), \quad \text { where } i \notin f n(\mathcal{E}) \\
& \left.\left.\langle\mathcal{E}[\operatorname{spawnAt} q M]\rangle_{p} \longrightarrow_{d} \nu i .\left(\langle\mathcal{E}[\operatorname{return} i]\rangle_{p}\left|i\{\}_{p}\right|\langle M\rangle\right\rangle=\operatorname{rput} i\right\rangle_{q}\right), \quad \text { where } i \notin \operatorname{fn}(\mathcal{E}) \\
& \langle\mathcal{E}[\text { rput } i M]\rangle_{p} \mid i\{\}_{q} \longrightarrow_{d}\langle\mathcal{E}[\text { return }()]\rangle_{p} \mid i\{M\}_{q} \\
& \langle\mathcal{E}[\text { rput } i M]\rangle_{p} \mid i\{N\}_{q} \longrightarrow_{d}\langle\mathcal{E}[\text { return }()]\rangle_{p} \mid i\{N\}_{q} \\
& \langle\mathcal{E} \text { [get } i]\rangle_{p}\left|i\{M\}_{p} \longrightarrow_{d}\langle\mathcal{E}[\operatorname{return} M]\rangle_{p}\right| i\{M\}_{p} \\
& \left\langle\mathcal{E}\left[\text { dist } q_{1} q_{2}\right]\right\rangle_{p} \longrightarrow{ }_{d}\left\langle\mathcal{E}\left[\text { return } d\left(q_{1}, q_{2}\right)\right]\right\rangle_{p} \\
& \left.\langle\mathcal{E} \text { [equiDist } r]\rangle_{p} \longrightarrow_{d}\langle\mathcal{E} \text { [return } M]\right\rangle_{p}, \quad \text { where } M \text { is an equidistant basis for the ball } D(p ; r)
\end{aligned}
$$

Figure 5. Small-step semantics of HdpH.

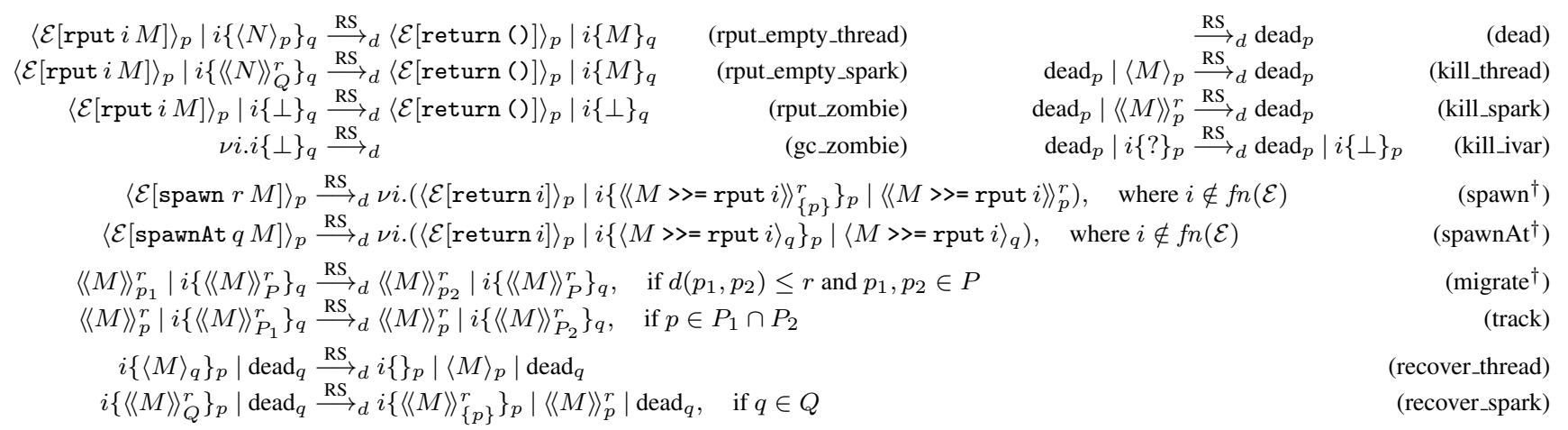

Figure 6. Additional rules for small-step semantics of HdpH-RS; rules marked with $\dagger$ replace eponymous HdpH rules.

spark/thread in the empty IVar $i$. Rule (migrate) works similarly as in $\mathrm{HdpH}$ except for ensuring that the supervising IVar $i$ continues to track the migrating spark, i.e. the new location of the spark remains a member of the tracking set $P$. That set may change via rule (track) in arbitrary ways, provided the current location of the supervised spark remains a member, modelling the supervisor's changing and uncertain knowledge about the location of a supervised spark.

The final two rules model the recovery of tasks that have been lost due to faults. A thread supervised by IVar $i$ on $p$ and executing on dead node $q$ is replicated on $p$, after which $i$ ceases to supervise as there is no point supervising a thread on the same node. A spark supervised by IVar $i$ on $p$ and known to reside on some node in the tracking set $Q$ is replicated on $p$ if any node $q \in Q$ is dead; afterwards $i$ continues to supervise, now tracking $\{p\}$, the location of the replica spark. Due to the inherent uncertainty of tracking, sparks may be replicated even when actually residing on healthy nodes.

\subsection{Relating Fault Tolerant and Fault Oblivious Semantics}

In order to relate the $\mathrm{HdpH}$ and $\mathrm{HdpH}-\mathrm{RS}$ semantics, we need to compare their respective normal forms. In $\mathrm{HdpH}$, thread $\langle N\rangle_{p}$ is a normal form of state $S$, denoted $S \downarrow_{d}\langle N\rangle_{p}$, iff $S \longrightarrow_{d}^{*}\langle N\rangle_{p}$ and $\langle N\rangle_{p}$ is irreducible or $N=$ return (). We have defined normal forms of arbitrary states $S$, yet we will mostly be interested in normal forms of threads $\langle M\rangle_{p}$ with $f n(M)=\emptyset$, as these threads correspond to tasks spawned and potentially replicated. Note that the restriction $f n(M)=\emptyset$ makes $\langle M\rangle_{p}$ a root thread guaranteeing well-formedness of normal forms, hence precluding normal forms being stuck (up to divergence).

Before defining HdpH-RS normal forms, we note that in any $\stackrel{\mathrm{RS}}{\longrightarrow} d$ reduction, rule (dead) permutes with every rule to the left. Consequently, we ban rule (dead) and instead start reduction from states of the form $S \mid \operatorname{dead}_{P}$, where $P=\left\{p_{1}, \ldots, p_{n}\right\}$ is a set of nodes and $\operatorname{dead}_{P}$ is short for $\operatorname{dead}_{p_{1}}|\ldots| \operatorname{dead}_{p_{n}}$.

In HdpH-RS, thread $\langle N\rangle_{p}$ is a normal form of state $S$, written $S \downarrow_{d}^{\mathrm{RS}}\langle N\rangle_{p}$, iff there is a set $P$ such that $S\left|\operatorname{dead}_{P} \stackrel{\mathrm{RS}^{*}}{d}\langle N\rangle_{p}\right|$ $\operatorname{dead}_{P}$ and $\langle N\rangle_{p}$ is irreducible or $N=\operatorname{return}($ ). Moreover, $\langle N\rangle_{p}$ is a failure-free normal form of $S$, denoted $S \downarrow_{d}^{\mathrm{FF}}\langle N\rangle_{p}$, if it satisfies the above definition with $P=\emptyset$. It is immediate that reductions leading to a failure-free normal form cannot use any of the rules (kill_*), (recover_*) and (gc_zombie).

We can prove that $\mathrm{HdpH}$ and $\mathrm{HdpH}-\mathrm{RS}$ agree on normal forms in the absence of failures.

Lemma 1. Let $M$ be a term with $\mathrm{fn}(M)=\emptyset$. Then for all terms $N$ and nodes $p,\langle M\rangle_{p} \downarrow_{d}\langle N\rangle_{p} \Leftrightarrow\langle M\rangle_{p} \downarrow_{d}^{F F}\langle N\rangle_{p}$.

Proof sketch. The bisimilarity between HdpH and HdpH-RS reductions is obvious, except for the rules governing spark migration. For the forward direction a (track) transition must be inserted before and after every (migrate) transition; for the reverse direction it suffices to delete all (track) transitions.

We aim to transform reductions with failures into failure-free reductions, preserving normal forms. This isn't possible in general; it does require some restriction on the use of location information. Let $M$ be a term with $f n(M)=\emptyset$. We call $M$ location-invariant iff it does not matter where it executes, that is $\langle M\rangle_{p} \downarrow_{d}\langle N\rangle_{p} \Leftrightarrow$ 
$\langle M\rangle_{q} \downarrow_{d}\langle N\rangle_{q}$, for all terms $N$ and nodes $p$ and $q$. We call $M$ transitively location-invariant iff for all nodes $p$ and all root threads $\langle N\rangle_{q}$ reachable from $\langle M\rangle_{p}, N$ is location-invariant.

Now we can prove that the failure-free normal forms of transitively location-invariant terms are exactly their HdpH-RS normal forms.

Lemma 2. Let $M$ be a term with $f n(M)=\emptyset$. If $M$ is transitively location-invariant then for all terms $N$ and nodes $p,\langle M\rangle_{p} \downarrow_{d}^{R S}$ $\langle N\rangle_{p} \Leftrightarrow\langle M\rangle_{p} \downarrow_{d}^{F F}\langle N\rangle_{p}$.

Proof sketch. The reverse direction is trivial. For the forward direction, construct a failure-free reduction from a HdpH-RS reduction by induction on the number of (recover-*) rules. For each replicated spark, the failure-free reduction mimics the migration and execution of the successful replica, i. e. the replica that eventually filled the spark's IVar. For each replicated thread, the failure-free reduction mimics the execution of the successful replica, yet translated to the node $q$ to which the original thread was spawned (ignoring that $q$ is supposed to be dead); thanks to location-invariance this translation does not affect the normal form that is eventually written to the thread's IVar.

Combining lemmas 1 and 2, we find that, for transitively location-invariant terms at least, $\mathrm{HdpH}$ and $\mathrm{HdpH}-\mathrm{RS}$ agree on the normal forms.

Theorem 3. Let $M$ be a term with $f n(M)=\emptyset$. If $M$ is transitively location-invariant then for all terms $N$ and nodes $p,\langle M\rangle_{p} \downarrow_{d}$ $\langle N\rangle_{p} \Leftrightarrow\langle M\rangle_{p} \downarrow_{d}^{R S}\langle N\rangle_{p}$.

Observations. Firstly, inspecting the proof sketch of Lemma 2. it is obvious that location-invariance is not actually required of all reachable root threads but only of the ones arising from spawnAt. Thus the precondition of Theorem 3 could be weakened. In particular, location-invariance could be dropped completely for the sublanguage that restricts task distribution to spawn only.

Secondly, for the purpose of presenting a simple semantics, we have ignored all observable effects apart from locations, and location-invariance took care of reconciling the effects with task replication. A DSL with more realistic effects (e.g. tasks performing IO) would have to take more care. On top of locationinvariance, effects would need to be idempotent, i. e. invariant under replication, in order to guarantee semantic equivalence between $\mathrm{HdpH}$ and $\mathrm{HdpH}-\mathrm{RS}$ programs.

Finally, $\mathrm{HdpH}$ and $\mathrm{HdpH}-\mathrm{RS}$ are non-deterministic in general as decisions taken by the non-deterministic scheduler may become observable, e.g. in case migrating tasks call myNode (defined in Section 3.2. The sublanguage that restricts task distribution to spawnAt only is deterministic, due to entirely deterministic scheduling. Whether there are more interesting deterministic sublanguages, in the face of truly non-deterministic scheduling, is an interesting and timely [16] open question.

\section{Validating the HdpH-RS Scheduler}

Due to the various sources of non-determinism in faulty distributed systems it is easy to make mistakes in their correctness arguments, hence the need for validation by model checking and testing.

Replication is a common fault tolerance technique, for example in the Erlang supervisor behaviour [19] and Hadoop [34]. In both Erlang and Hadoop, tasks are placed only once, simplifying replication and recovery. In contrast, the $\mathrm{HdpH}-\mathrm{RS}$ scheduler must consider spark migration when identifying replication candidates.

HdpH-RS Promela abstraction. The unbounded state space of the HdpH-RS scheduler is abstracted as a finite state Promela model. The Promela abstraction models node failure, and the la-

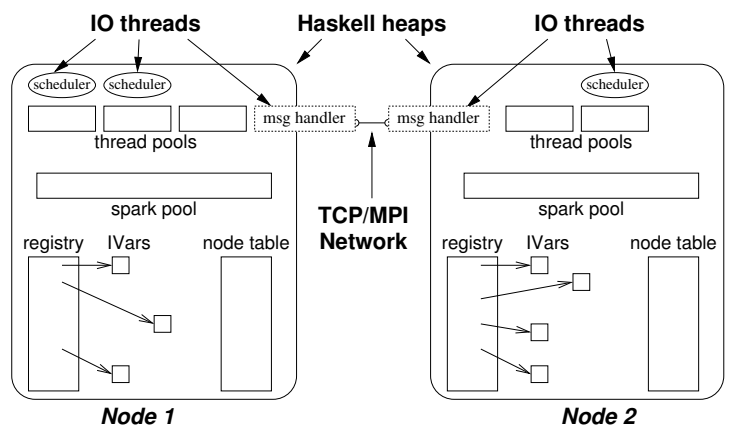

Figure 7. Runtime system architecture of $\mathrm{HdpH}$ and $\mathrm{HdpH}-\mathrm{RS}$.

tencies of work stealing and failure detection in the network abstraction layer (Section 6.1).

The model only validates the supervision of a spark created with spawn; threads placed by spawnAt cannot migrate and hence are a far simpler to supervise. The model includes one immortal supervising node, three mortal work stealing nodes, one spark and its corresponding initially empty future. The work stealing routines on the supervisor and three thieves are translated to a finite automaton, incorporating the six additional RTS messages needed for reliable work stealing (Section 6.3). If the supervisor detects a node failure that may eliminate the spark from the abstract machine state, it adds a replica to its local sparkpool, honouring the (recover_spark) rule from Section 4 Any node that holds a spark replica may at any time transmit a value into the empty future hosted on the supervisor. The model is described in full in [30], the Promela code is available [31.

Verification with SPIN. A key property of the HdpH-RS design is to guarantee the evaluation of supervised sparks, as recorded by filling the corresponding IVar on the supervising node. We model this by defining ivar_full to be a Boolean that becomes true when the future hosted on the supervisor is filled. Now we can specify in linear temporal logic that this variable is always eventually true, i. e. $\diamond \square$ ivarfull. The property is true despite arbitrary combinations of node failures, provided that the supervising node itself does not fail. Checking this property increases our confidence that the elaborate HdpH-RS work stealing protocol outlined in Section 6.3 correctly implements the semantics in Section 4 and in particular the rules (migrate) and (track).

The SPIN model checker exhaustively searches the model's state space to validate that the property holds in all states. SPIN explores 22.4 million transitions to a reachable depth of 124 transitions, proving that none of the 8.2 million reachable states violate the property.

Chaos Monkey testing. Besides model checking an abstraction, fault injection [13] was used on a suite of benchmarks (Section 8) to test the resilience of HdpH-RS in the presence of multiple random failures. Tests compare failure-free $\mathrm{HdpH}$ runs with $\mathrm{HdpH}-\mathrm{RS}$ runs in the presence of random failures. All tests pass [30].

\section{Implementation}

\subsection{RTS architecture}

Figure 7 depicts the key data structures and threads that make up the shared $\mathrm{HdpH}$ and $\mathrm{HdpH}-\mathrm{RS}$ RTS architecture. As the RTS is implemented in Haskell, the data structures are concurrently mutable maps, tables and queues in the Haskell heap, and the threads are Haskell IO threads. Each node runs several scheduler IO threads, typically one per core, and a message handler IO thread. 
Each scheduler owns a thread pool, a concurrent double-ended queue storing threads, i. e. computations of type Par (). The back end of the queue is only accessible to the owner, both for enqueueing, e. g. after unblocking, and for retrieving threads. The front end of the queue is accessible to all schedulers for stealing threads, similar to [27]. The message handler also owns a thread pool. However, unlike the schedulers, the message handler never retrieves threads; its threads must be stolen by a scheduler in order to be executed.

$\mathrm{HdpH}$ maintains one spark pool per node, a distance-indexed set of concurrent double-ended queues for storing sparks, i. e. serialisable computations of type Closure $(\operatorname{Par}())$. For the role of the spark pool in scheduling see Section 6.2

IVars are write-once buffers that are either empty or full, where empty IVars may also store a list of blocked threads to support the blocking get primitive as in [27], and a record of the thread or spark that is supposed to fill the IVar, to support task replication in HdpH-RS (Section 6.3).

$\mathrm{HdpH}$ maintains a registry per node, providing globally unique handles to locally hosted IVars, in order to support remote writing via rput. The registry is a concurrent map linking handles to their underlying IVars as detailed in [23].

For scalability, $\mathrm{HdpH}$ avoids a central table of all nodes. Instead, each node maintains its own node table, which is a distanceindexed set of some other nodes that it knows about. At system startup, the node table is initialised so that it holds random equidistant bases $Q_{r}$, one per distance $r$. The primitive equiDist returns exactly these $Q_{r}$. The node table also records individual nodes $q_{r}$, one per distance $r$, that have recently scheduled work to this node.

Two communication backends have been developed for $\mathrm{HdpH}$. The first provides MPI-based message passing for HPC architectures. Failures are fatal as MPI aborts on discovering faults.

The second backend targets COTS architectures and uses network-transport, a TCP-based network abstraction layer (NAL) designed for distributed Haskells such as CloudHaskell [8]. The NAL provides connection-oriented communication primitives that simplify message passing and fault detection. In particular, the NAL generates connection-lost events which indicate potential remote node failure. The backend reacts to these events and eventually, after failing to re-establish a lost connection over a period of time, propagates DEADNODE messages to the HdpH-RS scheduler.

\subsection{Topology Aware Scheduling}

Rule (migrate) in Section 4.1 models topology aware scheduling non-deterministically, constrained by the distance metric. This section details aspects of HdpH's topology aware work stealing algorithm, including its task selection policy. When a node $p_{0}$ executes the call spawn $r$ task, the task is converted into a spark (which involves creating and registering an IVar for the result) and added to the spark pool queue for distance $r$.

When $p_{0}$ runs out of work, and its own spark pool is non-empty, it uses the following local spark selection policy: Pick a spark with minimal radius; if there are several such sparks, pick the one at the back of the queue, i. e. the youngest or most recently stolen spark. Thus, $\mathrm{HdpH}$ prioritises sparks with small radii for local scheduling. As an aside, local scheduling requires to unClosure the spark, thereby converting it into a thread.

If, on the other hand, $p_{0}$ runs out of work with its own spark pool empty then it will engage in a distributed work stealing protocol comprising the messages FISH, SCHEDULE and NOWORK. In fact $p_{0}$ does not wait for its spark pool to drain completely; to hide latency work stealing is initiated as soon as the spark pool hits a low water mark.

Figure 8 illustrates the protocol with a successful and an unsuccessful stealing attempt. In both cases, $p_{0}$ starts by sending a FISH message to a random node nearby, that is a minimal distance away.

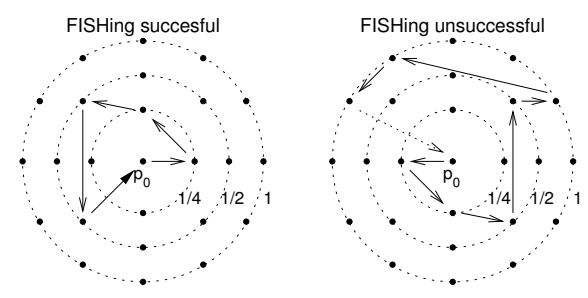

Figure 8. HdpH topology aware work stealing protocol.

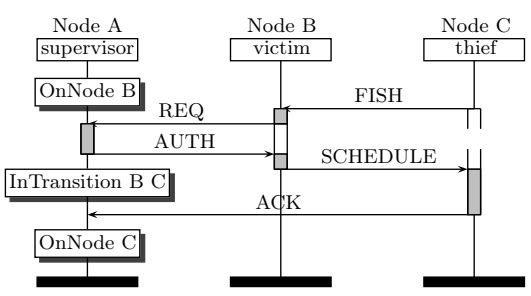

Figure 9. Fault tolerant work stealing protocol in $\mathrm{HdpH}-\mathrm{RS}$.

When a node $p$ receives a FISH message originating from $p_{0}$, it tries to find a suitable spark using the following remote spark selection policy: Pick a spark with minimal radius from the set of sparks whose radius is greater or equal to $d\left(p, p_{0}\right)$; if there are several such sparks, pick the one at the front of the queue, i. e. the oldest one. Thus for remote scheduling, $\mathrm{HdpH}$ prioritises sparks whose radii match the distance to the node requesting work. If remote spark selection is successful, $p$ sends a SCHEDULE message containing the selected spark $M$ and its radius $r$ to $p_{0}$. On receipt of SCHEDULE from $p, p_{0}$ inserts $M$ into the spark pool queue for distance $r$, and records $p$ in the node table at distance $r$. If remote spark selection is unsuccessful, $p$ forwards the FISH message to a random node $q$ such that $d\left(q, p_{0}\right)=d\left(p, p_{0}\right)$ or $d\left(q, p_{0}\right)>d\left(p, p_{0}\right)$. That is, the FISH message slowly "ripples away" from its originating node, as illustrated in Figure 8 , how slowly depends on how often the message is forwarded to a node at the same distance. If there is no work the FISH will eventually be forwarded to a node $q$ such that $d\left(q, p_{0}\right)=1$ is maximal. To stop the FISH bouncing forever, it will only be forwarded further a fixed number of times, 2 in Figure 8 after which a NOWORK message is sent to $p_{0}$. Upon receipt of NOWORK, $p_{0}$ backs off for some milliseconds before initiating another work stealing attempt.

The node table is used to select the targets for a forwarded FISH. Before $p$ forwards a FISH originating from $p_{0}$ it decides whether to increase the distance $r=d\left(p, p_{0}\right)$ i. e. whether to ripple further out. If not, $p$ forwards to a random node of the equidistant basis $Q_{r}$ recorded in its node table. If the distance is increased (minimally) to $r^{\prime}>d\left(p, p_{0}\right)$ then $p$ forwards to the node $q_{r^{\prime}}$ recorded in its node table as recent source of work at distance $r^{\prime}$, if it exists, otherwise to a random node of the equidistant basis $Q_{r^{\prime}}$. Thus, the work stealing protocol combines random searching for work with targeted probing of recent sources, while prioritising stealing from nearby.

\subsection{Fault Tolerant Scheduling}

The fault tolerance implementation in HdpH-RS is threefold. First, the HdpH scheduler is extended to track task locations. Second, the structure of IVars are extended to fulfil the role of supervised futures. Third, the scheduler replicates potentially lost tasks when failures are detected.

Reliable scheduling extension. The fault tolerant work stealing protocol is illustrated with a message sequence chart in Figure 9 
The protocol involves the supervisor in spark migration, and six additional RTS messages are used in HdpH-RS for the purpose of supervised work stealing. A thief targets a victim with a FISH message. If the victim has a sufficient number of sparks, then it sends a request to the supervisor as a REQ message for it be scheduled to the thief. The location state recorded by a supervisor for a spark is either OnNode or InTransition. The supervisor checks that the spark's location is marked as OnNode. If it is, an AUTH message is returned to the victim. Otherwise, a DENIED message is returned. When the supervisor and victim is the same node i.e. the spark is on the supervisor, the REQ and AUTH messages by-pass the network layer (Section 6.1). Instead, local function calls are used to determine the response to a FISH message.

Replica counts are used to avoid race conditions when multiple replicas co-exist. Only the spark tagged with the highest replica number may be scheduled elsewhere. The response to a REQ message regarding an older replica is an OBSOLETE message. A node that receives an OBSOLETE reply will discard the spark and send a NOWORK message to the thief.

Supervised futures. The spawn and spawnAt HdpH-RS primitives create extended versions of IVars to store additional state for fault tolerance. A copy of the task closure is held within the empty IVar, in case replication is later necessary. The location of the corresponding spark or thread, either OnNode $(p)$ or InTransition $(p, q)$, is stored in the IVar, together with a replica number counting how often the spark or thread has been replicated. A flag indicating whether to schedule the task lazily or eagerly is also stored in the IVar.

A spark created with spawn in HdpH-RS is transmitted as a tuple consisting of the following three components: the task to be evaluated, the task replica number, and a global handle to the IVar that will receive the task's result. The replica number and IVar handle are included in REQ and ACK messages to allow the supervisor to update the location state of the corresponding IVar.

Task replication. Task location state is used in the recovery phase to ensure that lost tasks are replicated. If failure is reported, i.e. a $\operatorname{DEADNODE}(p)$ message is received from the transport layer (Section 6.1, then the state of all empty IVars in the registry is inspected to identify replication candidates. A task is replicated in either of two cases. First, when its location record is OnNode $(p)$, indicating that it was on the dead node at the point of failure. Second, when its location record is $\operatorname{InTransition}(p, q)$ or $\operatorname{InTransition}(q, p)$, indicating that the task was in-flight either towards or away from the dead node.

This pessimistic replication strategy may lead to multiple copies of a spark. A migrating spark may survive a node failure, provided it was stolen from the failed node in time. Hence, an obsolete spark may be executed and its result written to the IVar. Assuming idempotence, this scenario is indistinguishable from the one where the obsolete spark has been lost.

The replication of sparks conforms to the (recover_spark) rule in Section 4 If a spark is to be re-scheduled, the replica count in the IVar is incremented. Then a new spark, consisting of the stored task, replica number and IVar handle, is added to the supervisor's spark pool, from where it may be stolen once again. The replication of threads is simpler, and conforms to the (recover_thread) rule. Rescheduling a thread is done by adding the stored task to the thread pool of the supervisor's message handler.

\section{Algorithmic Skeletons}

$\mathrm{HdpH}$ skeletons provide high-level coordination abstractions and are implemented using the primitives of Section 3 These abstractions provide topology awareness or fault tolerance depending on whether they are run by the topology aware scheduler of $\mathrm{HdpH}$,

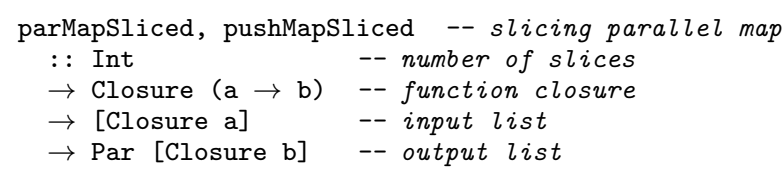

parMapReduceRangeThresh, pushMapReduceRangeThresh -- dEc :: Closure Int -- threshold

$\rightarrow$ Closure (Int,Int) -- range to divide/compute over

$\rightarrow$ Closure (Closure Int $\rightarrow$ Par (Closure a)) -- map fun

$\rightarrow$ Closure (Closure a $\rightarrow$ Closure a $\rightarrow$ Par (Closure a))

$\rightarrow$ Closure a -- initial value for reduction

$\rightarrow$ Par (Closure a) -- mapreduced result

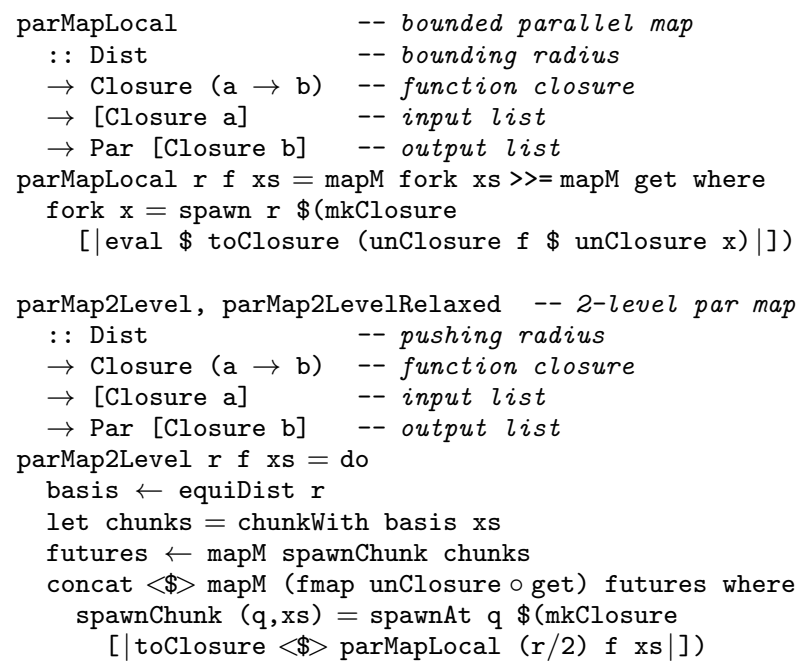

Figure 10. Some HdpH skeleton APIs and implementations.

or the reliable scheduler of HdpH-RS. HdpH and HdpH-RS provide libraries with around 30 skeletons, including several divideand-conquer, map/reduce, parallel map, and parallel buffer variants [25, 30]. Figure 10 outlines a selection of skeletons used in the evaluation in Section 8 .

Topology agnostic skeletons make no use of the $\mathrm{HdpH}$ distance primitives ${ }^{5}$ Four such skeletons are used in the evaluation of HdpH-RS (Section 8.3. The skeletons parMapSliced and pushMapSliced divide the input list into a given number of slices and evaluate each slice in parallel. For example, dividing the list $\left[e_{1}, \ldots, e_{5}\right]$ into three slices yields a list $\left[\left[e_{1}, e_{4}\right],\left[e_{2}, e_{5}\right],\left[e_{3}\right]\right]$ and three parallel tasks that are distributed lazily by parMapSliced or eagerly in a round-robin fashion by pushMapSliced.

Two divide-and-conquer skeletons are used to implement Mandelbrot in Section 8.3, again with both lazy and eager task placement. The skeletons generalise the parMapReduceRangeThresh skeleton of the Par monad library [27] to distributed memory. The skeletons combine a map over a finite range, which is recursively split until its size falls under a threshold, with a binary reduction of the map results. Task placement relies on work stealing for parMapReduceRangeThresh, whereas tasks are eagerly pushed to random nodes with pushMapReduceRangeThresh. In HdpHRS these skeletons create a nested supervision tree that reflects the divide-and-conquer call tree.

Topology aware skeletons exploit the $\mathrm{HdpH}$ distance primitives to control locality by (1) restricting work stealing to nearby nodes,

${ }^{5}$ The HdpH distance primitives can be used in HdpH-RS but HdpH-RS assumes the topology to be discrete. 
e. g. parMapLocal (Figure 10) creates tasks bounded by radius $r$, resulting in a lazy distribution of work to nodes at most distance $r$ from the caller; and (2) eagerly spreading tasks to distant nodes across the system. For example parMap2Level uses a combination of eager and lazy work distribution. After obtaining an equidistant basis for radius $r$, it splits the input list into chunks, one per basis node, taking into account the size information present in the basis, and eagerly spawns a big task per basis node. This achieves quick distribution of big tasks across the architecture. Eagerly evaluating their big tasks with parMapLocal, each basis node becomes a local coordinator: spawning small tasks to be evaluated in their vicinity, i. e. at a distance of no more than $r / 2$. Thanks to equidistance of the basis nodes, the bounding radius of $r / 2$ guarantees that small tasks cannot stray too far.

A variant of this two-level skeleton, parMap2LevelRelaxed, differs only in relaxing the bound imposed on small tasks from $r / 2$ to $r$. The effect is to allow the stealing of small tasks even between previously isolated local coordinators, which can help mitigate imbalances in task distribution arising from irregular parallelism. Due to the work stealing algorithm's preference for local work (Section 6.3), stealing due to the relaxation is a last resort, and occurs mostly in the final stages of a computation when work is drying up.

All topology aware skeletons provide a semi-explicit interface for tuning of locality via a single distance parameter, without ever exposing locations. This abstract locality control is intended to facilitate performance portability between parallel architectures. By not exposing locations these skeletons are location-invariant in the sense of Section 4.3 so their semantics won't change when switching from $\mathrm{HdpH}$ to $\mathrm{HdpH}-\mathrm{RS}$, although the performance is likely to change. We conjecture that not exposing locations also guarantees that these skeletons hide the effects of non-deterministic scheduling and compute deterministic results.

\section{Evaluation}

Benchmark platforms. HdpH and HdpH-RS are evaluated on HECToR and a COTS Beowulf cluster using the appropriate communication backends. HECToR is the UK's publicly funded HPC platform with a total of $90 \mathrm{~K}$ cores; it comprises 2816 compute nodes, each with 32 AMD Opteron cores at $2.3 \mathrm{GHz}$ sharing $32 \mathrm{~GB}$ of RAM, divided into 4 NUMA regions. The 256 core Beowulf cluster comprises 32 nodes connected via Gigabit Ethernet; each node has $12 \mathrm{~GB}$ of memory and 8 Intel Xeon cores at $2 \mathrm{GHz}$.

Benchmarks applications. We evaluate scaling and topology awareness of $\mathrm{HdpH}$ on two version of the SumEuler benchmark (Sections 8.1 and 8.2 and on a computational algebra case study (Section 8.4). Scaling and fault tolerance of HdpH-RS is evaluated on the Mandelbrot and Summatory Liouville benchmarks (Section 8.3). The benchmarks typically compare several coordination alternatives like distributing work lazily/eagerly, being topology aware/agnostic, or being fault tolerant/oblivious. The sources of $\mathrm{HdpH}$ and $\mathrm{HdpH}-\mathrm{RS}$, including benchmark applications, are publicly available [22, 30].

\subsection{Scaling}

We investigate the weak scaling of HdpH from 1 to 1024 HECToR nodes (i.e. from 32 to $32 \mathrm{~K}$ cores) using the moderately irregular SumEuler benchmark, a data-parallel computation of the sum of Euler's $\varphi$ function over an integer interval. This benchmark relies on GAP to compute $\varphi$, and each HECToR node is populated with 31 GAP instances, coordinated by one $\mathrm{HdpH}$ instance. Distributed coordination is performed by the parMap2Level and parMap2LevelRelaxed skeletons, and the topology is discrete, i. e. the distance between $\mathrm{HdpH}$ instances is always 1 .

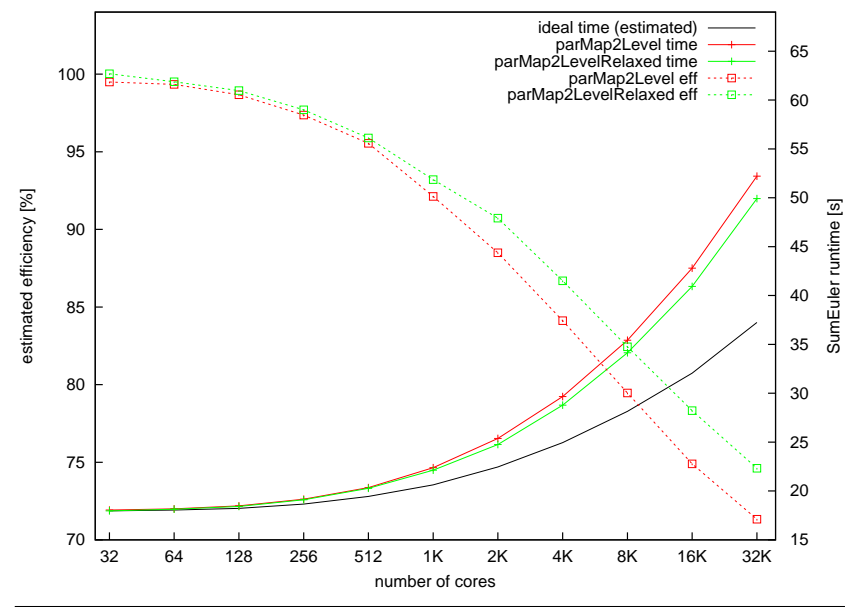

Figure 11. SumEuler — weak scaling up to $32 \mathrm{~K}$ cores.

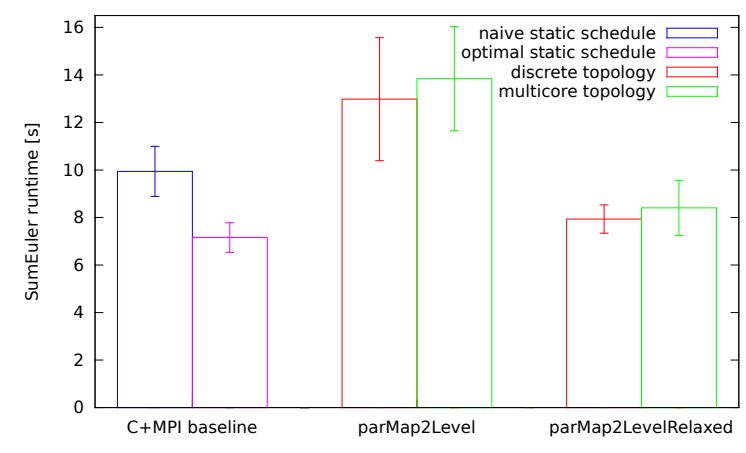

Figure 12. SumEuler - impact of topology on 256 cores.

Figure 11 shows weak scaling and efficiency results. The input interval starts at 6.25 million integers on one HECToR node (32 cores), and doubles when doubling the number of cores up to 6.4 billion integers on 1024 nodes (32K cores). Doubling the size of the input interval more than doubles the amount of work as computing $\varphi$ is more expensive on larger numbers, so we estimate a runtime curve for perfect scaling (by sampling and interpolating the runtimes of small tasks). The runtime graphs in Figure 11 show that the two skeletons do not scale perfectly. However, even on $32 \mathrm{~K}$ cores their runtimes are still within a factor of 1.5 of the ideal.

Efficiency (i.e. speedup divided by number of cores) is estimated by relating the observed runtimes to the (estimated) perfect scaling time. The graphs show that efficiency is steadily declining, yet remains above $70 \%$ even on $32 \mathrm{~K}$ cores. These graphs also show that parMap2LevelRelaxed offers a small efficiency advantage over parMap2Level.

\subsection{Topology Awareness}

The impact of different topologies and of different modes of task placement on $\mathrm{HdpH}$ performance are also investigated with the SumEuler benchmark. Yet, here $\varphi$ is computed naively in $\mathrm{HdpH}$ rather than relying on GAP. Coordination is again performed by the skeletons parMap2Level and parMap2LevelRelaxed, both with radius 1 . The experiments are performed on the 256 core Beowulf with either the discrete topology, or the standard multicore topology (i.e. distance between cores sharing memory is $\frac{1}{2}$ ).

Figure 12 shows runtimes, averaged over 11 runs, and $95 \%$ confidence intervals. Sequential runtime is $1115 \pm 20$ seconds. 


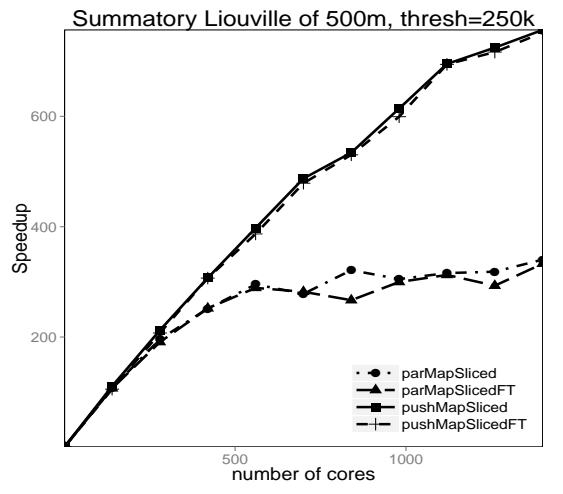

Figure 13. Summatory Liouville speedup on HECToR.

The skeletons divide the input into 1024 tasks, so average task granularity is about 1 second, but varies by 3 orders of magnitude, between 2 seconds and a few milliseconds. We observe that, as in the weak scaling experiment, parMap2LevelRelaxed performs best, with speedups of 130 to 140, whereas parMap2Level only achieves speedups of 80 to 90 . Remarkably, the topology does not matter; the multicore topology appears to perform slightly worse but the overheads stay well within the error margin. We conclude that a 256-core cluster is too small to suffer from locality issues.

Figure 12 also compares the performance of $\mathrm{HdpH}$ to a baseline SumEuler benchmark, implemented natively in $C+M P I$. Sequential runtime of the $\mathrm{C}$ code is $956 \pm 1$ seconds, about $15 \%$ faster than Haskell. A naive static MPI task placement achieves speedups of about 95; the optimal static schedule (found by experiment) yields speedups of about 130 . Ultimately, C+MPI with optimal static scheduling is about 10 to $15 \%$ faster than $\mathrm{HdpH}$ with parMap2LevelRelaxed, matching the sequential performance gap. This shows that (1) HdpH introduces minimal overheads, and (2) HdpH work stealing can compete with optimal static scheduling for this benchmark.

\subsection{Fault Tolerance}

A total of five benchmarks are used to measure scalability, supervision overheads, and recovery overheads of HdpH-RS in the thesis [30].

Scaling and supervision overheads. The speedup of the Summatory Liouville program outlined in Section 3.1 is measured on HECToR up to 1400 cores using $[20,40 . .200]$ nodes with $n=500 m$ and a threshold of $250 k$. This generates 2000 tasks so that all PEs may be saturated with at least one task up to 1400 cores with ideal scheduling.

Figure 13 compares the performance of the slicing parallel map skeletons parMapSliced and pushMapSliced (Section 7), both with reliable scheduling enabled (indicated by suffix FT) and disabled. Beyond 280 cores, the eager skeletons outperform the lazy ones, reaching peak speedups of around 750 versus 340 . More importantly, however, we observe that the FT graphs stay close to the graphs of their unreliable cousins, that is the overhead of reliable scheduling is negligible.

Fault recovery costs. The $\mathrm{HdpH}-\mathrm{RS}$ scheduler is designed to survive both single and simultaneous node failures. The cost of recovering from such failures is assessed with the well-known Mandelbrot benchmark. The coordination is performed by the divideand-conquer skeletons par/pushMapReduceRangeThresh (Section 7), generating 1023 tasks.

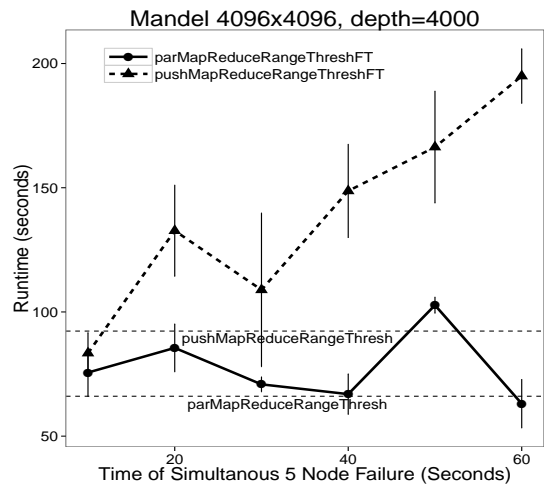

Figure 14. Simultaneous node failures (Mandelbrot on Beowulf).

For each skeleton the mean duration of five fault-free runs, 66 and 92 seconds respectively, is plotted as a horizontal base line in Figure 14 To assess recovery time, 5 nodes are killed simultaneously after $10,20, \ldots, 60$ seconds. Each recovery experiment is performed 5 times, and Figure 14 plots the average recovery runtimes (including standard error bars). The recovery overheads for parMapReduceRangeThresh are consistently low, and variability limited. Recovery overheads for pushMapReduceRangeThresh increase over time, and variability is generally higher. These results highlight a preference towards lazy on-demand scheduling to minimise recovery time.

\subsection{Representation Theory Case Study}

This section briefly reports the performance of $\mathrm{HdpH}$ coordinating GAP on a case study [24] from the representation theory of Hecke algebras [11]. Given generators $M_{1}, \ldots, M_{m}$, square matrices of polynomials in $\mathbb{Z}\left[x, x^{-1}\right]$, the problem is to find a (non-trivial) symmetric matrix $Q$ over $\mathbb{Z}\left[x, x^{-1}\right]$ such that the product of $Q$ with each generator is itself symmetric. Depending on the Hecke type $\mathrm{E}_{m}(m=6,7,8)$, the dimension of the generators and the degrees of the polynomials in $Q$ may vary considerably.

We parallelise the three most time-consuming phases of the algorithm for finding $Q$ : (1) solving of homomorphic images over finite fields, (2) solving of interpolation problems over rationals, and (3) final product symmetry check over polynomial matrices. All algebraic computations are done by sequential GAP instances and coordinated by $\mathrm{HdpH}$, as in Section 8.1 Some illustrative results are as follows. For medium-size $\mathrm{E}_{7}$ representations (23 to 38) we obtain relative speedups of between 40 and 55 using 106 GAP instances on 16 Beowulf nodes (128 cores). For small $\mathrm{E}_{8}$ representations (11 to 15 ) we obtain relative speedups of between 116 and 548 using 992 GAP instances on 32 HECToR nodes (1024 cores).

\section{Discussion}

Large commodity manycore architectures will have high failure rates and a non-uniform communication topology between cores. We have outlined the design of a pair of shallowly embedded Haskell DSLs, HdpH and HdpH-RS, to address these challenges for computations with irregular parallelism (Section 3). We have presented operational semantics for both DSLs and established conditions for semantic equivalence (Section 4). We have briefly sketched validation of the sophisticated work stealing protocol of HdpH-RS by model checking and testing (Section 5). We have described the DSL implementations, focusing on how the work stealing schedulers achieve topology awareness and fault tolerance (Section 6). We have provided examples of algorithmic skeletons, 
including skeletons for sophisticated topology aware work distribution (Section 7). An initial evaluation using 5 benchmarks on a Beowulf cluster and the HECToR HPC platform shows good weak scaling of $\mathrm{HdpH}$ up to $32 \mathrm{~K}$ cores, and that $\mathrm{HdpH}-\mathrm{RS}$ has low overheads both in the presence and absence of faults. In a computational algebra case study we obtain speedups of up to 548 coordinating 992 GAP instances on 1024 cores (Section 8).

Although developed for symbolic computation the HdpH DSLs are general purpose, being designed to manage dynamic and irregular task parallelism on large scale hierarchical architectures. They cope well with complex algorithms, coordination patterns, and data structures, but typical numeric HPC workloads are not well suited. The $\mathrm{HdpH}$ programming model works particularly well where tasks are stateless. For good performance, task execution time should greatly outweigh communication time, which is largely determined by the size of the closures transmitted, hence Big Data workloads with large memory footprints are also not suitable. As HdpH-RS retains backups of supervised closures, its performance is additionally predicated on a small retained closure footprint. That is, either the number of supervised closures is small, or the closures are small in size (on average). Thus HdpH-RS offers a trade-off between fault tolerance and memory use.

Currently, HdpH and HdpH-RS provide orthogonal features. An immediate engineering task is to amalgamate topology awareness and fault tolerance into a single DSL. While HdpH was designed for architectures with $10^{5}$ cores, we only have made systematic measurements up to $32 \mathrm{~K}$ cores for pragmatic reasons: access to all $90 \mathrm{~K}$ cores of HECToR is simply too expensive. As COTS and HPC platforms grow, we expect that larger architectures will eventually become more affordable, which would help us continue to use $\mathrm{HdpH}$ for solving open problems in algebraic representation theory.

\section{Acknowledgments}

The work was funded by EPSRC grants HPC-GAP (EP/G05553X), AJITPar (EP/L000687/1) and Rathlin (EP/K009931/1), and EU grant RELEASE (FP7-ICT 287510). The authors thank Lilia Georgieva, Sam Lindley, Daria Livesey, Greg Michaelson, Jeremy Singer and the anonymous referees for helpful feedback.

\section{References}

[1] J. Allen. Effective Akka. O'Reilly, 2013.

[2] J. Armstrong, R. Virding, C. Wikström, and M. Williams. Concurrent Programming in ERLANG. Prentice Hall, 2nd edition, 1996.

[3] L. A. Barroso, J. Clidaras, and U. Hölzle. The Datacenter as a Computer. Morgan \& Claypool, 2nd edition, 2013.

[4] R. D. Blumofe and P. A. Lisiecki. Adaptive and reliable parallel computing on networks of workstations. In USENIX 1997 Annual Technical Conference, Anaheim, CA, USA, 1997.

[5] P. B. Borwein, R. Ferguson, and M. J. Mossinghoff. Sign changes in sums of the Liouville function. Mathematics of Computation, 77(263): 1681-1694, 2008.

[6] F. Cappello. Fault tolerance in petascale/exascale systems. Int. Journal HPC Applications, 23(3):212-226, 2009.

[7] M. M. T. Chakravarty, R. Leshchinskiy, S. L. Peyton Jones, G. Keller, and S. Marlow. Data parallel Haskell: a status report. In DAMP 2007, Nice, France, pages 10-18. ACM, 2007.

[8] J. Epstein, A. P. Black, and S. L. Peyton-Jones. Towards Haskell in the cloud. In Haskell 2011, Tokyo, Japan, pages 118-129. ACM, 2011.

[9] A. Foltzer et al. A meta-scheduler for the Par-monad: composable scheduling for the heterogeneous cloud. In ICFP 2012, Copenhagen, Denmark, pages 235-246. ACM, 2012.

[10] GAP Group. GAP - groups, algorithms, and programming, 2007. http://www.gap-system.org
[11] M. Geck and J. Müller. James' conjecture for Hecke algebras of exceptional type, I. J. Algebra, 321(11):3274-3298, 2009.

[12] R. H. Halstead Jr. Multilisp: A language for concurrent symbolic computation. ACM Trans. Prog. Lang. Syst., 7(4):501-538, 1985.

[13] T. Hoff. Netflix: Continually test by failing servers with Chaos Monkey. http://highscalability.com December 2010.

[14] V. Janjic and K. Hammond. Granularity-aware work-stealing for computationally-uniform Grids. In CCGrid 2010, Melbourne, Australia, pages 123-134. IEEE, 2010.

[15] V. Kravtsov, P. Bar, D. Carmeli, A. Schuster, and M. T. Swain. A scheduling framework for large-scale, parallel, and topology-aware applications. J. Parallel Distrib. Comput., 70(9):983-992, 2010.

[16] L. Kuper, A. Turon, N. R. Krishnaswami, and R. R. Newton. Freeze after writing: Quasi-deterministic parallel programming with LVars and handlers. In POPL 2014, San Diego, USA. ACM, 2014.

[17] J. Lifflander, S. Krishnamoorthy, and L. V. Kale. Work stealing and persistence-based load balancers for iterative overdecomposed applications. In HPDC'12, Delft, The Netherlands, pages 137-148. ACM, 2012.

[18] S. Linton et al. Easy composition of symbolic computation software using SCSCP. J. Symb. Comput., 49:95-119, 2013.

[19] M. Logan, E. Merritt, and R. Carlsson. Erlang and OTP in Action. Manning, 2010.

[20] R. Loogen, Y. Ortega-Mallén, and R. Peña-Marí. Parallel functional programming in Eden. J. Funct. Program., 15(3):431-475, 2005.

[21] W. Ma and S. Krishnamoorthy. Data-driven fault tolerance for work stealing computations. In ICS 2012, Venice, Italy, pages 79-90. ACM, 2012.

[22] P. Maier and R. Stewart. HdpH source code, 2014. https:// github.com/PatrickMaier/HdpH

[23] P. Maier and P. Trinder. Implementing a high-level distributedmemory parallel Haskell in Haskell. In IFL 2011, Lawrence, KS, USA, Revised Selected Papers, LNCS 7257, pages 35-50. Springer, 2012.

[24] P. Maier, D. Livesey, H.-W. Loidl, and P. Trinder. High-performance computer algebra: A Hecke algebra case study. In Euro-Par 2014, Porto, Portugal. Springer, 2014. To appear.

[25] P. Maier, R. Stewart, and P. W. Trinder. Reliable scalable symbolic computation: The design of SymGridPar2. Computer Languages, Systems \& Structures, 40(1):19-35, 2014.

[26] S. Marlow, S. L. Peyton-Jones, and S. Singh. Runtime support for multicore Haskell. In ICFP 2009, Edinburgh, Scotland, pages 65-78. ACM, 2009 .

[27] S. Marlow, R. Newton, and S. L. Peyton-Jones. A monad for deterministic parallelism. In Haskell 2011, Tokyo, Japan, pages 71-82. ACM, 2011.

[28] S.-J. Min, C. Iancu, and K. Yelick. Hierarchical work stealing on manycore clusters. In PGAS 2011, Galveston Island, TX, USA, 2011.

[29] S. L. Peyton-Jones, A. Gordon, and S. Finne. Concurrent Haskell. In POPL 1996, St. Petersburg Beach, USA, pages 295-308, 1996.

[30] R. Stewart. Reliable Massively Parallel Symbolic Computing: Fault Tolerance for a Distributed Haskell. PhD thesis, Heriot-Watt University, 2013.

[31] R. Stewart. Promela abstraction of HdpH-RS reliable scheduler extension, 2013. https://raw.github.com/robstewart57/ phd-thesis/master/spin_model/hdph_scheduler.pml

[32] P. W. Trinder et al. GUM: A portable parallel implementation of Haskell. In PLDI 1996, Philadelphia, USA, pages 79-88. ACM, 1996.

[33] P. W. Trinder et al. Algorithms + Strategy = Parallelism. J. Funct. Program., 8(1):23-60, 1998.

[34] T. White. Hadoop - The Definitive Guide: MapReduce for the Cloud. O'Reilly, 2009.

[35] G. Wrzesinska, R. van Nieuwpoort, J. Maassen, and H. E. Bal. A simple and efficient fault tolerance mechanism for divide-and-conquer systems. In CCGrid 2004, Chicago, USA, pages 735-734. IEEE, 2004. 